\title{
Concept Design of a Miniaturized Abdominal Aorta Sensing Stent to Prevent Renal Impairment in Heart Failure Patients
}

\author{
Samah Atiyat ${ }^{1}$, Shadi Karabsheh ${ }^{2}$ \\ ${ }^{1}$ University of Alabama at Birmingham \\ Birmingham, Alabama, USA \\ Samahaa@UAB.edu \\ ${ }^{2}$ Cardiology Department, King Faisal Specialist Hospital \& Research Center \\ AI-Rawdhah, Jeddah, Saudi Arabia \\ SKarabsheh@KFSHRC.edu.sa
}

\section{Extended Abstract}

Demographics of heart failure (HF) patients show that they represent age 65 years and older. Heart Failure Society of America (HFSA) confirmed that the HF has the highest hospital readmission rates, high rate of physician visits each year, and more than half of them die within five years of diagnosis [1]. Aging is associated with increasing comorbidities specifically cardiac and kidney disorders due to multifactorial including hemodynamic alterations and neurohormonal activation [2]. This condition contributes to increased mortality and difficult recovery. Having an insight into the blood flow and pressure helps to correlate the mechanical conditions with the pathological changes [3]. In addition, the level of serum creatinine in the blood is a useful guide to kidney function estimated as the glomerular filtration rate (GFR). Biosensors provide an accurate and rapid assessment of small-scaled hemodynamic as an alternative to the time consuming, complicated and expensive laboratory tests, magnetic resonance imaging, and ultrasound, which can further help in preventing renal or cardio impairment [4].

Abdominal aorta (AA) is the part of the aorta that passes through the diaphragm and extending down to the abdomen [5]. Renal arteries branch from the AA. Minimally invasive implantation of a biosensor close to that region is typical for accurate detection of any possible hemodynamic alterations thus cardio or renal deterioration. Endovascular stenting procedures are performed in the AA [6][7]. Some of the FDA-approved and commercially available stent graft devices include Medtronic, Gore, Endologix, and Cook. These currently marketed stents are available in various shapes and sizes in terms of diameter and length.

The proposed concept design incorporates an AA stent, close upon the renal arteries branch region, minimum in length $(<8 \mathrm{~mm})$ to abate platelet reactivity, with optimal scaffolding wall opposition to maintain unrestricted flow through the aorta. Fabricated with biocompatible materials such as cobalt chromium $(\mathrm{CoCr})$ or Platinum Chromium (PtCr). With a strut thickness of $\sim 70 \mu \mathrm{m}$. The stent structure functions as a wireless telemetry antenna which facilitate the viability of continuous monitoring. The scope of this design is to embed multiparameter biosensors into the stent to accurately assess AA hemodynamic. The biosensors system consists of a piezo-resistive pressure sensor, flow sensor, and serum creatinine sensor. Pressure and flow sensors are well developed and commercially available [8]. The serum creatinine biosensor enzymatically detects creatinine level in the blood in a similar way of glucose sensors.

By continuously monitoring the hemodynamic along with the creatinine level, both cardiac output and estimated GFR could be calculated and analyzed using an entrenched battery-less ASIC providing real-time evaluation of renal and cardio functionality.

\section{References}

[1] N. Filipovic, M. Ivanovic, D. Krstajic, and M. Kojic, "Hemodynamic flow modeling through an abdominal aorta aneurysm using data mining tools.," IEEE Trans. Inf. Technol. Biomed., vol. 15, no. 2, pp. 189-194, 2011.

[2] H. Yu, L. Ai, M. Rouhanizadeh, D. Patel, E. S. Kim, and T. K. Hsiai, "Flexible polymer sensors for in vivo intravascular shear stress analysis," J. Microelectromechanical Syst., vol. 17, no. 5, pp. 1178-1186, 2008.

[3] J. Park, J. K. Kim, S. A. Park, D. S. Sim, M. H. Jeong, and D. W. Lee, “3D-printed biodegradable polymeric stent 
integrated with a battery-less pressure sensor for biomedical applications," in TRANSDUCERS 2017 - 19th International Conference on Solid-State Sensors, Actuators and Microsystems, 2017, pp. 47-50.

[4] Y. Peng, T. Wang, W. Jiang, X. Liu, X. Wen, and G. Wang, "Modeling and optimization of inductively coupled wireless bio-pressure sensor system using the design of experiments method," IEEE Trans. Components, Packag. Manuf. Technol., vol. 8, no. 1, pp. 65-72, 2018.

[5] C. P. Cheng, R. J. Herfkens, and C. A. Taylor, "Comparison of abdominal aortic hemodynamics between men and women at rest and during lower limb exercise," J. Vasc. Surg., vol. 37, no. 1, pp. 118-123, 2003.

[6] B. John et al., "Telemetric system for monitoring of endoleak in abdominal aorta aneurysm using multiple pressure sensors integrated on a stent graft," in Proceedings - 2016 IEEE Biomedical Circuits and Systems Conference, BioCAS 2016, 2017, pp. 384-387.

[7] V. Isailovic et al., "Computer model and clinical relevance of abdominal aorta aneurysm with compliant nonlinear material wall," in Proceedings of the IEEE/EMBS Region 8 International Conference on Information Technology Applications in Biomedicine, ITAB, 2010.

[8] L. Yu, B. J. Kim, and E. Meng, "Chronically implanted pressure sensors: Challenges and state of the field," Sensors (Switzerland), vol. 14, no. 11. pp. 20620-20644, 2014. 